\title{
Surgical video on the sternotomy-sparing medial approach thyroidectomy for a substernal multinodular goiter case report
}

\author{
Allen Young ${ }^{1}$, Harry Ching ${ }^{1}$, Sabrina $\mathrm{Ho}^{2}$, Robert C. Wang ${ }^{1}$ \\ ${ }^{1}$ Department of Otolaryngology Head and Neck Surgery, University of Nevada Las Vegas, Las Vegas, NV, USA; ${ }^{2}$ School of Medicine, University of \\ Nevada Las Vegas, Las Vegas, NV, USA \\ Correspondence to: Allen Young, MD, MPH. University of Nevada Las Vegas, 1701 West Charleston Boulevard Suite 490, Las Vegas, NV 89102, USA. \\ Email: AllenHYoung@gmail.com.
}

\begin{abstract}
Substernal goiters can significantly alter the native anatomy of patients and require a different approach than normal, benign goiters. Its enlarged bulk and substernal extension can present a major technical challenge for surgeons with increased risk of recurrent laryngeal nerve injury, parathyroid glands injury, and possible need for sternotomy or thoracostomy to fully excise the substernal component. A sternotomy or thoracotomy approach is associated with a significant surge in complications such as increased intraoperative blood loss, mediastinitis, hematoma, wound infection, osteomyelitis, chest bone fracture, and sternal dehiscence. On this video presentation, we visually demonstrate our medial approach thyroidectomy technique to facilitate transcervical resection of a large substernal goiter without the need for sternotomy. A 33-year-old female with dyspnea on exertion and orthopnea was found to have a massive 9-cm goiter compressing the trachea and extending below the aortic arch. She underwent a total thyroidectomy and en bloc delivery of the substernal component with successful preservation of bilateral recurrent laryngeal nerves and parathyroid glands. There was minimal deep neck and substernal dissection and no sternotomy was necessary. Postoperatively, her parathyroid hormone and calcium levels were normal and she was discharged on postoperative day (POD) 2. On follow-up, she had no hoarseness, dysphagia, or hypocalcemia. A medial approach thyroidectomy promotes early exposure of the recurrent laryngeal nerve and safe transection of Berry's ligament allowing mobilization of the thyroid and transcervical delivery of substernal components without injury of the native anatomic structures. Furthermore, this technique serves to minimize the morbidity and mortality associated with sternotomy, thus reducing hospital stay and improving patient satisfaction.
\end{abstract}

Keywords: Substernal goiter (SSG); case report; medial approach thyroidectomy; thyroid goiter; thyroidectomy technique

Received: 28 September 2020; Accepted: 18 December 2020; Published: 20 April 2022.

doi: 10.21037 /jovs-20-160

View this article at: http://dx.doi.org/10.21037/jovs-20-160

\section{Introduction}

Substernal goiter (SSG) has an incidence ranging from $0.2 \%$ to $0.45 \%$ of the general population depending the specific definition used (1). Two commonly used definitions are a goiter with any portion of its mass extending below the thoracic inlet or a goiter with more than half its volume below the thoracic inlet (2). Compression of the trachea, esophagus, and great vessels may produce symptoms of dyspnea, orthopnea, sleep disorder, dysphagia, and visible neck disfigurement (3). Thyroidectomy for large SSGs must be approached in a different manner than normal, benign goiters due to their large bulk and substernal extension, which significantly alters native anatomic structures. This presents a technical challenge for surgeons as excessive dissection of a massive SSG can cause substantial damage to the parathyroid glands and the recurrent laryngeal nerve (RLN) (2). Risk of RLN injury with thyroid goiters have 


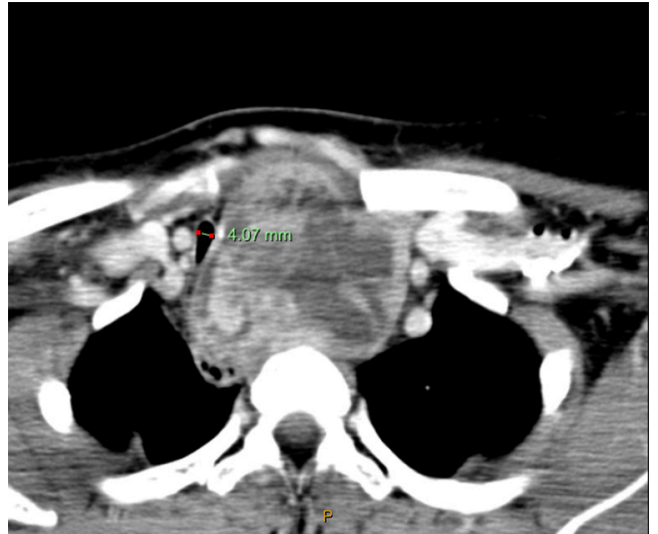

Figure 1 CT neck axial view of large substernal thyroid goiter with compression of trachea to $4.07 \mathrm{~mm}$. P, posterior; CT, computerized tomography.

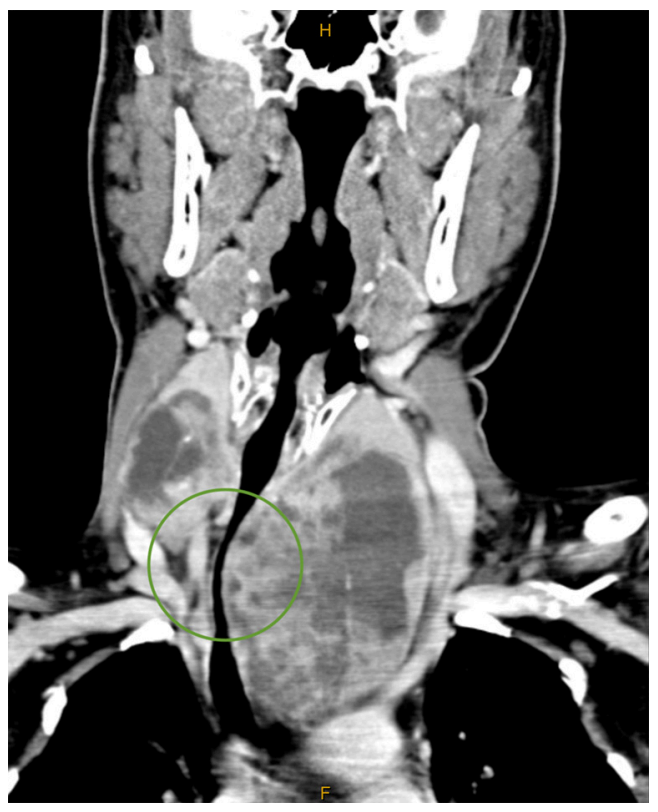

Figure 2 CT neck coronal view of large substernal thyroid goiter below the thoracic inlet and abutting the aortic arch. Green circle: area of tracheal narrowing. H, towards head; F, towards feet; CT, computerized tomography.

been as high as $17.5 \%$ (4). Total or partial sternotomy has been necessary in $1-11 \%$ of patients with SSG $(5,6)$, incurring severe morbidity such as increased blood loss, mediastinitis, hematoma, wound infection, osteomyelitis, chest bone fracture, and sternal dehiscence (7). To reduce the significant morbidity risks associated with extensive dissection and sternotomy, we present a video of our sternotomy-sparing medial-approach thyroidectomy technique for massive SSGs with early visualization and preservation of the RLNs and minimal deep neck and mediastinal dissection (8).

We present the following article in accordance with the CARE reporting checklist (available at https://jovs. amegroups.com/article/view/10.21037/jovs-20-160/rc).

\section{Case presentation}

All procedures performed in this study were in accordance with the ethical standards of the institutional research committee and with the Helsinki Declaration (as revised in 2013). Written informed consent was obtained from the patient for publication of this case report and accompanying images. A copy of the written consent is available for review by the editorial office of this journal.

A 33-year-old female with no prior medical history presents with a large thyroid mass had associated severe orthopnea requiring sleeping upright and dysphagia. Computerized tomography (CT) of the neck showed a substantial $9 \mathrm{~cm}$ left greater than right thyromegaly compressing the trachea to $3.95 \mathrm{~mm}$ and extending substernally below the aortic arch (Figures 1,2). Ultrasoundguided biopsy showed benign cytologic findings consistent with a large substernal thyroid goiter. She underwent total thyroidectomy using a medial approach with minimal deep neck and substernal dissection. A thoracic surgeon was available on standby, but thoracotomy was not needed. The surgery was performed by the senior author (RC Wang) with the full understanding and written consent of the patient. A flowchart of our surgical technique is shown in Figure 3.

A horizontal neck incision is made and subcutaneous flaps are raised superiorly to the hyoid bone and inferiorly to the sternal notch. Pre-laryngeal midline tissues are exposed and the pyramidal lobe resected if present. The strap muscles are elevated off the thyroid with the sternothyroid muscle transected as needed to gain exposure (Video 1, 1:16-1:30). The thyroid isthmus is divided (Video 1, 1:30-1:43). The large right thyroid lobe without substernal extension was approached first. The inferior and superiormedial portions of Berry's ligament were divided, which allowed reflection of the right lobe superiorly and medially, leading to the identification of the right RLN in a usual lateral and inferior approach (Video 1, 1:45-2:09). The nerve is then traced to its entrance into the larynx and vessels are ligated on the surface of the thyroid gland to preserve 


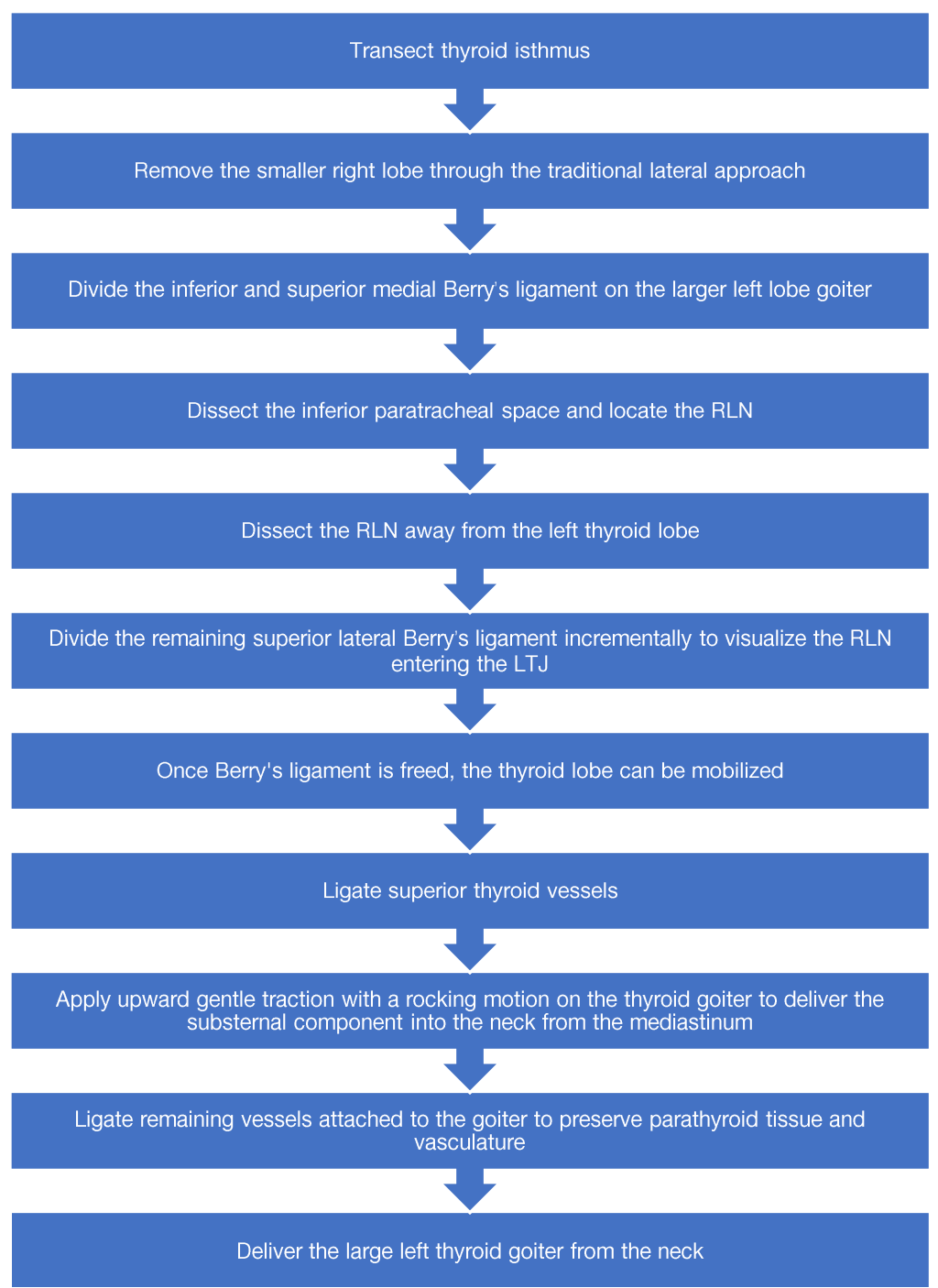

Figure 3 Flowchart of surgical steps for sternotomy-sparing medial approach thyroidectomy. RLN, recurrent laryngeal nerve; LTJ, laryngotracheal junction.

parathyroid tissue and vascular supply (Video 1, 2:10-2:14). The remaining superior-lateral Berry's ligament is divided and the right lobe delivered off the field (Video 1, 2:16-2:28).

The larger left lobe with substernal extension must be approached differently due to the lateral and inferior approaches not being feasible. After the superior-medial and all of the inferior portions of Berry's ligament are divided, the inferior paratracheal space is dissected and the RLN is identified. The nerve is then traced superiorly and inferiorly as well as dissected away from the thyroid gland (Video 1, 2:39-3:18). The remaining superior-lateral Berry's ligament is divided incrementally as the RLN is exposed (Video 1, 3:19-3:53). Once all the tracheal attachments to the thyroid gland are freed, the thyroid is able to be mobilized, allowing the superior pole to be delivered into the field and the superior thyroid vessels ligated. Upward gentle traction with a rocking motion, if needed, is applied to the goiter, which allows the substernal component to be delivered into the neck from the mediastinum (Figure 4) with ligation of remaining vessels close to the gland to preserve parathyroid tissue (Video 1, 3:54-4:15). The skin incision is closed in a multilayered fashion with absorbable sutures.

The patient tolerated the procedure well. Her postoperative parathyroid hormones and ionized calcium levels were within normal limits and she required no calcium supplementation. She displayed no voice changes 


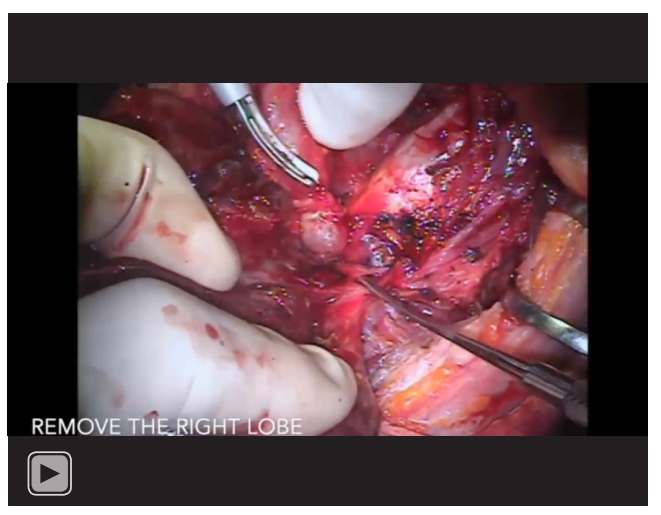

Video 1 Sternotomy-sparing surgical dissection technique of a large thyroid substernal goiter with preservation of bilateral recurrent laryngeal nerves and parathyroid glands.

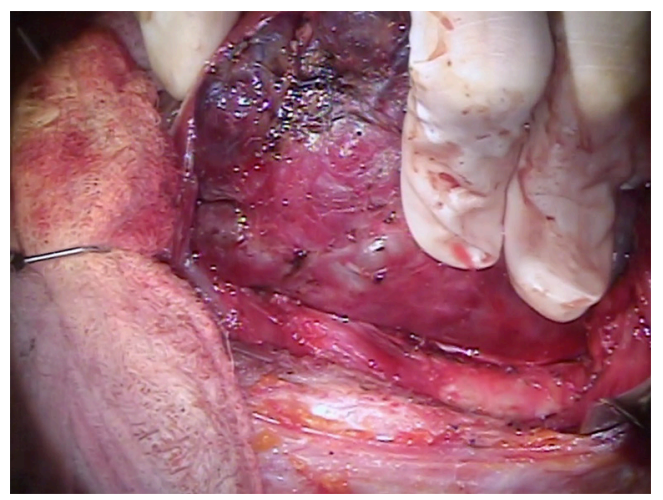

Figure 4 Upward retraction on the thyroid goiter to help deliver the goiter from the thoracic cavity.

nor hypocalcemic symptoms. She was discharged on postoperative day (POD) 2 tolerating a regular diet without dysphagia. Final pathology showed benign goiter weighing 270 grams total. Outpatient follow up on POD 14 showed a well-healing neck incision with intact bilateral vocal cord motion on flexible laryngoscopy. The patient reports resolution of prior dysphagia and orthopnea. She states she is now able to sleep comfortably without any compressive symptoms over her neck. Furthermore, with the resolution of her bulky neck appearance, she is no longer embarrassed to attend public events. She was pleased with the decision to surgically remove her goiter.

\section{Discussion}

The majority of SSG originate from the downward extension of the thyroid along the cervical and mediastinal fascial planes. SSGs have presented a technical challenge to surgeons throughout history as the surgical strategy differs from that used for small benign goiters due to its massive size and distortion of native anatomy. In the 1920s, the popular technique was morcellation of the mass with subsequent intracapsular fragmentation and suction of material from within the mass (9). In 1993, Charles Proye published the toboggan approach which emphasized early identification of the RLN superiorly followed by sharp dissection inferiorly along a lateral approach (10). There was also the drawer maneuver which involves gripping the SSG with both hands and pulling it like a drawer out of the neck (9). Historical transcervical approaches include isolation of the superior pole of the thyroid, separation of the thyroid from the anterior trachea with lysis of Berry's ligament, and blunt finger dissection of the SSG (1).

Sternotomy or lateral thoracotomy has been utilized in $1-11 \%$ of SSG patients with all patients showing thyroid tissue below the aortic arch (5-7). Although most small SSGs can be resected with a single transcervical incision, traditional teaching has recommended sternotomy for cases of large SSGs extending beyond the aortic arch into the mediastinum (11). Further elucidation in the literature of patient factors that may necessitate sternotomy approach include (I) goiter too large to remove through thoracic inlet, (II) a mediastinal blood supply to the goiter, (III) goiter causing anatomic variations of the RLN and parathyroids, and (IV) venous congestion due to compromised drainage (11). The shape of the goiter (hourglass, iceberg, and dumbbell) and its mass continuity have not been significant predictors of which patients needed sternotomies (7). The decision to perform a sternotomy or lateral thoracic should not be taken lightly as it not only increases the postoperative risk for patents such as hemorrhage, mediastinitis, and wound dehiscence, but also requires more operative planning, resources, and prolonged hospitalization $(7,11)$.

This surgical video visually demonstrates the successful removal of a substantial $9 \mathrm{~cm}$ goiter through our sternotomy-sparing medial approach thyroidectomy. According to traditional teaching and studies in the literature, the patient would have been categorized to need a sternotomy due to the SSG extending beyond the aortic arch with severe distortion of the RLN and parathyroid glands. However, through our unique surgical technique, we were able to achieve early identification of the RLN, preservation of parathyroid vasculature, and release of the thyroid from its trachea attachments, which culminates in a 
safe and facile delivery of the goiter from the mediastinum.

\section{Conclusions}

The resection of large goiters with substernal extension is a technically demanding procedure with major risk of complications. Although rare, the use of sternotomies can further exacerbate the severity of postoperative complications and resource utilization. Our judicious sternotomy-sparing medial approach thyroidectomy can help facilitate early exposure of the RLN, safe division of Berry's ligament, and delivery of the substernal and deep neck components into the surgical field, minimizing patient morbidity and hospitalization.

\section{Acknowledgments}

This study was presented at the American Academy of Otolaryngology Head and Neck Surgery (AAO-HNS) 2019 Annual Meeting in September 2019 in New Orleans, LA, USA. Funding: None.

\section{Footnote}

Reporting Checklist: The authors have completed the CARE reporting checklist. Available at https://jovs.amegroups. com/article/view/10.21037/jovs-20-160/rc

Conflicts of Interest: All authors have completed the ICMJE uniform disclosure form (available at https://jovs. amegroups.com/article/view/10.21037/jovs-20-160/coif). The authors have no conflicts of interest to declare.

Ethical Statement: The authors are accountable for all aspects of the work in ensuring that questions related to the accuracy or integrity of any part of the work are appropriately investigated and resolved. All procedures performed in this study were in accordance with the ethical standards of the institutional research committee and with the Helsinki Declaration (as revised in 2013). Written informed consent was obtained from the patient for publication of this case report and accompanying images. A copy of the written consent is available for review by the editorial office of this journal.

Open Access Statement: This is an Open Access article distributed in accordance with the Creative Commons Attribution-NonCommercial-NoDerivs 4.0 International
License (CC BY-NC-ND 4.0), which permits the noncommercial replication and distribution of the article with the strict proviso that no changes or edits are made and the original work is properly cited (including links to both the formal publication through the relevant DOI and the license). See: https://creativecommons.org/licenses/by-nc-nd/4.0/.

\section{References}

1. Doulaptsi M, Karatzanis A, Prokopakis E, et al. Substernal goiter: Treatment and challenges. Twentytwo years of experience in diagnosis and management of substernal goiters. Auris Nasus Larynx 2019;46:246-51.

2. Hedayati N, McHenry CR. The clinical presentation and operative management of nodular and diffuse substernal thyroid disease. Am Surg 2002;68:245-51.

3. Shaha AR. Surgery for benign thyroid disease causing tracheoesophageal compression. Otolaryngol Clin North Am 1990;23:391-401.

4. Chang EHE, Kim HY, Koh YW, et al. Overview of robotic thyroidectomy. Gland Surg 2017;6:218-28.

5. Rugiu MG, Piemonte M. Surgical approach to retrosternal goitre: do we still need sternotomy? Acta Otorhinolaryngol Ital 2009;29:331-8.

6. White ML, Doherty GM, Gauger PG. Evidence-based surgical management of substernal goiter. World J Surg 2008;32:1285-300.

7. Nankee L, Chen H, Schneider DF, et al. Substernal goiter: when is a sternotomy required? J Surg Res 2015;199:121-5.

8. Ching HH, Kahane JB, Foggia MJ, et al. Medial Approach for the Resection of Goiters with Suprahyoid, Retropharyngeal, or Substernal Extension. World J Surg 2018;42:1415-23.

9. Hanson MA, Shaha AR, Wu JX. Surgical approach to the substernal goiter. Best Pract Res Clin Endocrinol Metab 2019;33:101312.

10. Proye CAG. Substernal goitres—surgical technique. Curr Pract Surg 1993;5:72-7.

11. Rui Sheng Y, Chong Xi R. Surgical approach and technique in retrosternal goiter: Case report and review of the literature. Ann Med Surg (Lond) 2015;5:90-2.

doi: 10.21037/jovs-20-160

Cite this article as: Young A, Ching $\mathrm{H}$, Ho S, Wang RC. Surgical video on the sternotomy-sparing medial approach thyroidectomy for a substernal multinodular goiter case report. J Vis Surg 2022;8:19. 